\title{
Finite-Time Tracking Control for a Class of MIMO Nonlinear Systems with Unknown Asymmetric Saturations
}

\author{
Fu Mingyu and Xu Yujie \\ College of Automation, Harbin Engineering University, Harbin 150001, China \\ Correspondence should be addressed to Xu Yujie; xuyujie@hrbeu.edu.cn
}

Received 27 May 2017; Revised 18 July 2017; Accepted 31 July 2017; Published 29 August 2017

Academic Editor: Quang Phuc Ha

Copyright (c) $2017 \mathrm{Fu}$ Mingyu and Xu Yujie. This is an open access article distributed under the Creative Commons Attribution License, which permits unrestricted use, distribution, and reproduction in any medium, provided the original work is properly cited.

\begin{abstract}
This paper addresses the problem of finite-time tracking control for multiple-input and multiple-output (MIMO) nonlinear systems with asymmetric saturations. A systematic approach is proposed to eliminate the effects of unmeasured external disturbances and unknown asymmetric saturations. In the proposed control strategy, a terminal sliding mode disturbance observer is provided to estimate the augmented disturbance (which contains the unknown asymmetric input saturation and external disturbance). The approximation error of the augmented disturbance can converge to zero in a fixed finite-time interval. Furthermore, a novel finitetime tracking control algorithm is developed to guarantee fast convergence of the tracking error. Compared with the existing results on finite-time tracking control, the chattering problem and the input saturation problem can be solved in a unified framework. Several simulations are given to demonstrate the effectiveness of the proposed approach.
\end{abstract}

\section{Introduction}

The convergence rate is an important issue for dynamical control systems in practice. To improve the control performance, the concept of finite-time stability has been proposed and investigated in several literatures [1-3]. Compared with the conventional Lyapunov asymptotical stability, the finitetime stability admits that the state of systems does not exceed a certain bound during a fixed finite-time interval [4]. Besides, robustness performances of the system can also be improved [5]. During the past decades, several finite-time control schemes have been proposed with the development of geometric homogeneity theory and finitetime Lyapunov stability theory [6-11]. In particular, terminal sliding mode control (TSMC) has been widely used in many practical control systems due to the robust enhanced ability.

As an effective finite-time control technique, TSMC has merits of handling control systems with large uncertainties, nonlinearities, and bounded external disturbances. In [12], a robust TSMC strategy was developed for rigid robotic manipulators in which an MIMO terminal switching plane variable vector was first defined and the relationship between the terminal switching plane variable vector and system error dynamics was established. In [13], a fast terminal dynamics was proposed in the design of the sliding mode control for single-input single-output nonlinear dynamical systems to improve the convergence rate during reaching and sliding phase. In $[14,15]$, nonsingular problem in TSMC was addressed. In [16], a fast nonsingular terminal sliding mode (FNTSM) was developed for piezoelectric actuators, which can avoid chatter problems. In [17], a terminal attractor with nonnegative exponential coefficient was introduced to generate a continuous FNTSM algorithm. In [18, 19], double exponential reaching law with variable coefficient was combined with FNTSM manifold to improve the convergence rate during reaching and sliding phase. More improved TSMC algorithms and their applications can be found in the literature [20-24]. All the aforementioned works have two drawbacks. (i) The upper boundary of external disturbances are assumed to be available. In order to guarantee the convergence of the system, large gain is chosen in front of the symbolic function, which may cause terrible twitter. (ii) The input saturation is not considered.

In order to eliminate chattering caused by the large gain, different methods have been developed. Some researchers 
proposed the high order sliding mode control [25, 26], while others introduced the disturbance observers into the control system [27-34]. In [25], the high order sliding mode controller was designed using integral sliding mode concept to achieve bounded uncertainties rejection. Based on previous work, a new higher order sliding mode controller with optimal reaching was designed in [26], which proves useful for the tuning of HOSM controller parameters. In [30-32], extended state observer was developed and has been widely used for its ease of implementation. In [32], a high-speed sliding mode observer (SMO) was proposed to estimate the rotor position and the angular velocity of the permanentmagnet synchronous motor (PMSM). In [35], a terminal sliding mode disturbance observer for SISO system has been studied to guarantee that the approximation error of disturbance observer converges to zero in finite time. And based on the geometric homogeneity, a novel distributed finitetime observer was presented for the followers to estimate the leader's velocity in [36]. Replacing the large gain with output of the disturbance observer will greatly mitigate the twitter, but the convergence of the closed-loop system needs further discussion.

Another problem in finite-time control is the input saturation, which may bring in chattering and degrade the control performance. In [37-39], the analysis and design of control systems with input saturation constraints have been studied. In [40], stability of the modified control system with saturation was further discussed. In [41], an auxiliary system was designed with the same order as that of the studied MIMO attitude system to handle the input saturation. Some adaptive type-1 or type- 2 fuzzy/neural schemes have also been proposed to control the nonlinear systems with actuator saturation in [42-44]. In [35], the input saturation was regarded as external disturbances, and a disturbanceobserver-based terminal sliding mode control was developed for SISO (single-input single-output) system. Most of the mentioned researches are based on the assumption that the input saturation is known. However, the actuator performance may be unknown in practice.

Inspired by the work of $[35,45]$, a disturbance-observerbased finite-time tracking control strategy is designed in this paper. But the difference is that our control plant is MIMO nonlinear system and input saturations are asymmetric. The effects of unknown asymmetric input saturation are combined with the external disturbance to form an augmented disturbance, which is estimated by a proposed terminal sliding mode disturbance observer. And the developed disturbance observer can guarantee that the augmented disturbance approximation error converges to zero in finite time. Then, a novel finite-time tracking control algorithm is developed for uncertain MIMO nonlinear systems. Compared with most existing research, our work has two main contributions: (1) the chattering problem and saturation problem are solved in a unified framework; (2) the finite-time convergence of all closed-loop state is guaranteed regardless of external disturbance and unknown asymmetric saturation. Thus, the proposed finite-time tracking controller actually can be applied to a large class of uncertain MIMO nonlinear systems.
The paper is organized as follows. The problem is formulated in Section 2, and some preliminary results of finite-time stability are also introduced. The detailed finite-time control scheme is presented in Section 3. Simulation examples are provided in Section 4. Finally, conclusions are drawn in Section 5.

\section{Problem Formulation}

2.1. Nonlinear System with Input Saturation. Consider the following uncertain nonlinear MIMO system:

$$
\begin{aligned}
\dot{\mathbf{x}}_{1} & =\mathbf{x}_{2}, \\
\dot{\mathbf{x}}_{2} & =\mathbf{f}(\mathbf{x})+\mathbf{g}(\mathbf{x}) \mathbf{u}+\mathbf{d}, \\
\mathbf{y} & =\mathbf{x}_{1},
\end{aligned}
$$

where $\mathbf{x}_{1}$ and $\mathbf{x}_{2}$ are the state of the system, $\mathbf{u}$ is the control input, $\mathbf{y}$ is the control output, $\mathbf{d}$ is the external disturbance, and $\mathbf{f}(\mathbf{x})$ and $\mathbf{g}(\mathbf{x})$ are continuously nonlinear functions. Here, $\mathbf{x}=\left[\begin{array}{ll}\mathbf{x}_{1} & \mathbf{x}_{2}\end{array}\right]^{T} \mathbf{x}_{1}, \mathbf{x}_{2}, \mathbf{y} \in \mathbb{R}^{n \times 1}, \mathbf{d} \in \mathbb{R}^{n \times 1}, \mathbf{u} \in \mathbb{R}^{m \times 1} \mathbf{f}(\mathbf{x}) \in$ $\mathbb{R}^{n \times 1}$, and $\mathbf{g}(\mathbf{x}) \in \mathbb{R}^{m \times n}$.

Due to the unknown asymmetric saturation constraints, the control input is given by the following form:

$$
\mathbf{u}= \begin{cases}\mathbf{u}_{\max } & \boldsymbol{v}>\mathbf{u}_{\max } \\ \boldsymbol{v} & -\mathbf{u}_{\min } \leq \boldsymbol{v} \leq \mathbf{u}_{\max } \\ -\mathbf{u}_{\min } & \boldsymbol{v}<-\mathbf{u}_{\min },\end{cases}
$$

where $\boldsymbol{v}$ is the designed control input command and $\mathbf{u}_{\max }$ and $\mathbf{u}_{\text {min }}$ are the unknown parameters of the control input saturation. Here, $\mathbf{u}_{\max } \neq \mathbf{u}_{\min }$ denotes the asymmetric saturation.

In practice, the performance of the actuators may change with different operating environment. For a marine vessel, the propulsive force of propeller is related to wind, current, and waves, which cannot be measured precisely. Thus, the input saturation is unknown to the controller designer. This situation may also occur for other plants.

2.2. Control Objective. In this paper, the control objective is to design a finite-time tracking controller for MIMO nonlinear system with disturbances and unknown asymmetric saturations. For the desired trajectory, the proposed terminal sliding mode control should guarantee finite-time convergence of the entire closed-loop signal. Then the control objective can be presented in the following mathematical form:

$$
\lim _{t \rightarrow T}\left\|\mathbf{y}-\mathbf{y}_{d}\right\|=0, \quad \text { for }-\mathbf{u}_{\min } \leq \mathbf{u} \leq \mathbf{u}_{\max } .
$$

And the desired trajectory $\mathbf{y}_{d}$ is assumed to be smooth, which means there exists a positive number $\Delta_{i}>0$, such that

$$
\left\|\mathbf{y}_{d}^{(i)}\right\| \leq \Delta_{i}, \quad i=1,2, \ldots, p .
$$

2.3. Finite-Time Lyapunov Stability. Before presenting the main results, an extended Lyapunov description of finite-time stability can be given by the following lemma. 


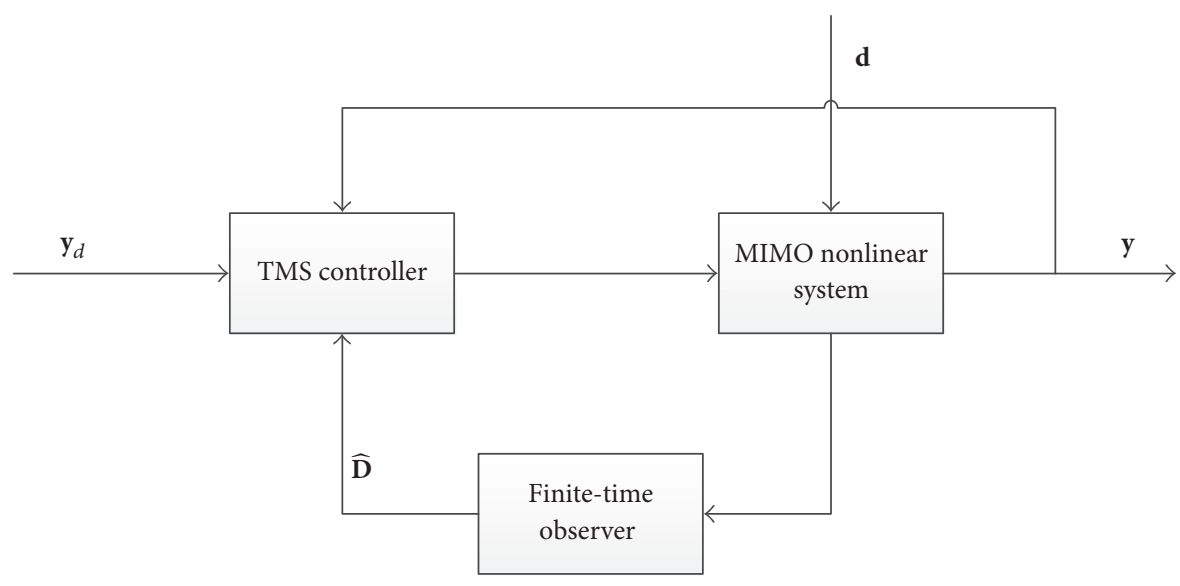

FIGURE 1: Framework of the control scheme.

Lemma 1 (see [46]). Assume that there exists a continuous positive definite function $V(t)$, which satisfies the following inequality:

$$
\dot{V}(t)+\alpha V(t)+\lambda V^{\gamma}(t) \leq 0, \quad \forall t>t_{0} .
$$

Then $V(t)$ converges to the equilibrium point in finite time $t_{s}$ :

$$
t_{s} \leq t_{0}+\frac{1}{\alpha(1+\gamma)} \ln \frac{\alpha V^{1-\gamma}\left(t_{0}\right)+\lambda}{\lambda},
$$

where $\alpha>0, \lambda>0$, and $0<\gamma<1$.

\section{Controller Design}

In this section, a finite-time disturbance observer is proposed to eliminate the chattering due to external disturbance and input saturation through feedforward compensation. Based on the output of the observer, the terminal sliding mode control for MIMO nonlinear system is developed, which can guarantee the finite-time convergence of the closed-loop system.

The control scheme can be represented by the framework in Figure 1. It should be noted that $\mathbf{d}$ is the external disturbance, while $\widehat{\mathbf{D}}$ denotes the estimated value which contains external disturbance and input saturation.

The control command is denoted by $v$ :

$$
\boldsymbol{\nu}=\mathbf{G}(\mathbf{x})^{-1} \boldsymbol{\nu}_{r},
$$

where $\boldsymbol{v}_{r}$ is the reference control command that will be given in the next section. And the control command error is defined as $\Delta \mathbf{u}=\mathbf{u}-\boldsymbol{v}$.

Substituting the control command into (1), the MIMO nonlinear system can be rewritten as

$$
\begin{aligned}
& \dot{\mathbf{x}}_{1}=\mathbf{x}_{2}, \\
& \dot{\mathbf{x}}_{2}=\mathbf{f}(\mathbf{x})+\mathbf{v}_{r}+\mathbf{D},
\end{aligned}
$$

where $\mathbf{D}=\mathbf{g}(\mathbf{x}) \Delta \mathbf{u}+\mathbf{d}$.
Since the upper and lower limits of the input saturation are unknown, the effect of $\mathbf{g}(\mathbf{x}) \Delta \mathbf{u}$ can be regarded as system uncertainty. Hence, the effect of external disturbance and asymmetric saturation can be rewritten into the form of augmented disturbance $\mathbf{D}$.

3.1. Finite-Time Disturbance Observer. In this section, a finite-time disturbance observer is developed from the terminal sliding mode observer to estimate the augmented disturbance $\mathbf{D}$. Firstly, we introduce a variable $\mathbf{z}$ to denote the estimation of $\mathbf{x}_{2}$. And the estimation error can be defined as follows:

$$
\mathbf{S}=\mathbf{z}-\mathbf{x}_{2}
$$

If the update law of $\mathbf{z}$ is given in the form of terminal sliding mode in (10), estimation error $\mathbf{S}$ can converge to zero in fixed time interval, which will be proved later.

$$
\dot{\mathbf{z}}=-\mathbf{K S}-\boldsymbol{\beta} \operatorname{sgn}(\mathbf{S})-\boldsymbol{\varepsilon} \mathbf{S}^{p / q}-\mathbf{F}(\mathbf{x}) \operatorname{sgn}(\mathbf{S})+\mathbf{v}_{r} .
$$

And the derivative of $\mathbf{S}$ can be calculated:

$$
\begin{aligned}
\dot{\mathbf{S}}= & \dot{\mathbf{z}}-\dot{\mathbf{x}}_{2} \\
= & -\mathbf{K S}-\boldsymbol{\beta} \operatorname{sgn}(\mathbf{S})-\boldsymbol{\varepsilon} \mathbf{S}^{p / q}-\mathbf{F}(\mathbf{x}) \operatorname{sgn}(\mathbf{S})-\mathbf{f}(\mathbf{x}) \\
& -\mathbf{D} .
\end{aligned}
$$

Then, the estimation of the augmented disturbance $\widehat{\mathbf{D}}$ can be given by

$$
\widehat{\mathbf{D}}=-\mathbf{K S}-\boldsymbol{\beta} \operatorname{sgn}(\mathbf{S})-\varepsilon \mathbf{S}^{p / q}-\mathbf{F}(\mathbf{x}) \operatorname{sgn}(\mathbf{S})-\mathbf{f}(\mathbf{x}),
$$

where $0<p<q, \mathbf{K}, \boldsymbol{\beta}$, and $\boldsymbol{\varepsilon}$ are positive definite diagonal matrices, and the parameters are chosen in the following form:

$$
\begin{aligned}
& \mathbf{K}=\operatorname{diag}\left(\left[\begin{array}{llll}
K_{1} & K_{2} & \cdots & K_{n}
\end{array}\right]\right), \\
& \boldsymbol{\beta}=\operatorname{diag}\left(\left[\begin{array}{llll}
\beta_{1} & \beta_{2} & \cdots & \beta_{n}
\end{array}\right]\right),
\end{aligned}
$$




$$
\begin{aligned}
& \boldsymbol{\varepsilon}=\operatorname{diag}\left(\left[\begin{array}{llll}
\varepsilon_{1} & \varepsilon_{2} & \cdots & \varepsilon_{n}
\end{array}\right]\right), \\
& \mathbf{F}(\mathbf{x})=\operatorname{diag}\left(\left[\left\|f(\mathbf{x})_{1}\right\|\left\|f(\mathbf{x})_{2}\right\| \cdots\left\|f(\mathbf{x})_{n}\right\|\right]\right), \\
& \mathbf{f}(\mathbf{x})=\left[\begin{array}{llll}
f(\mathbf{x})_{1} & f(\mathbf{x})_{2} & \cdots & f(\mathbf{x})_{n}
\end{array}\right]^{T}, \\
& \mathbf{D}=\left[\begin{array}{llll}
D_{1} & D_{2} & \cdots & D_{n}
\end{array}\right]^{T}, \\
& \mathbf{S}=\left[\begin{array}{llll}
S_{1} & S_{2} & \cdots & S_{n}
\end{array}\right]^{T}, \\
& \mathbf{S}^{p / q}=\left[\begin{array}{llll}
S_{1}^{p / q} & S_{2}^{p / q} & \cdots & S_{n}^{p / q}
\end{array}\right]^{T} .
\end{aligned}
$$

It is important to note that $\beta_{i}$ is the upper bound of $D_{i}$; that is, $\beta_{i}>\left|D_{i}\right|, i=1,2, \ldots, n$.

Here, we define the estimation error of the augmented disturbance $\widetilde{\mathbf{D}}=\widehat{\mathbf{D}}-\mathbf{D}$. Invoking (11)-(12), we can get

$$
\begin{aligned}
\widetilde{\mathbf{D}}= & -\mathbf{K S}-\boldsymbol{\beta} \operatorname{sgn}(\mathbf{S})-\boldsymbol{\varepsilon} \mathbf{S}^{p / q}-\mathbf{F}(\mathbf{x}) \operatorname{sgn}(\mathbf{S})-\mathbf{f}(\mathbf{x}) \\
& -\mathbf{D}=\dot{\mathbf{z}}-\dot{\mathbf{x}}_{2}=\dot{\mathbf{S}} .
\end{aligned}
$$

From the above formula, we can know that $\widetilde{\mathbf{D}}$ is related to the derivative of $\mathbf{S}$. If $\mathbf{S}$ converges to a fixed value, the estimation error of the augmented disturbance will converge to zero. Through derivation, $\mathbf{S}$ is finite-time stable and main results on the disturbance observer are presented in the following theorem.

Theorem 2. Consider the uncertain MIMO nonlinear system (8), if the finite-time disturbance observer is designed as (9)-(12), the augmented disturbance approximation error of the proposed terminal sliding mode disturbance observer will converge to zero in finite time.

Proof. Consider the Lyapunov function candidate:

$$
V_{1}=\frac{1}{2} \mathbf{S}^{T} \mathbf{S}
$$

Calculating the derivative of $V_{1}$, we obtain

$$
\begin{aligned}
\dot{V}_{1} & =\mathbf{S}^{T} \dot{\mathbf{S}}=\mathbf{S}^{T}\left(-\mathbf{K S}-\boldsymbol{\beta} \operatorname{sgn}(\mathbf{S})-\boldsymbol{\varepsilon} \mathbf{S}^{p / q}\right. \\
& -\mathbf{F}(\mathbf{x}) \operatorname{sgn}(\mathbf{S})-\mathbf{f}(\mathbf{x})-\mathbf{D})=-\sum_{i=1}^{n} K_{i} S_{i}^{2} \\
& -\sum_{i=1}^{n} \varepsilon_{i} S_{i}^{(p+q) / q}-\sum_{i=1}^{n}\left(\beta_{i}\left|S_{i}\right|+D_{i} S_{i}\right) \\
& -\sum_{i=1}^{n}\left(\left|f_{i}\right|\left|S_{i}\right|+f_{i} S_{i}\right) \leq-\sum_{i=1}^{n} K_{i} S_{i}^{2}-\sum_{i=1}^{n} \varepsilon_{i} S_{i}^{(p+q) / q} \\
& \leq-\lambda_{\min }(\mathbf{K}) \sum_{i=1}^{n} S_{i}^{2}-\lambda_{\min }(\boldsymbol{\varepsilon}) \sum_{i=1}^{n} S_{i}^{(p+q) / q} \\
& =-2 \lambda_{\min }(\mathbf{K}) V_{1}-2^{(p+q) / q} \lambda_{\min }(\boldsymbol{\varepsilon}) V_{1}^{(p+q) / 2 q}
\end{aligned}
$$

where $\lambda_{\min }(\mathbf{K})$ is the least eigenvalue of matrix $\mathbf{K}$.
From (16) and Lemma 1, we can conclude that the variable $\mathbf{S}$ will converge to zero in finite time. Assume that the initial moment is zero; then the convergence time of $\mathbf{S}$ can be calculated as follows:

$$
\begin{aligned}
t_{1}= & \frac{1}{2 \lambda_{\min }(\mathbf{K})(1+(p+q) / 2 q)} \\
& \cdot \ln \frac{2 \lambda_{\min }(\mathbf{K}) V_{1}^{(q-p) / 2 q}(0)+2^{(p+q) / q} \lambda_{\min }(\boldsymbol{\varepsilon})}{2^{(p+q) / q} \lambda_{\min }(\boldsymbol{\varepsilon})} .
\end{aligned}
$$

Due to the finite-time convergence of the variable $\mathbf{S}$, the estimation error of the augmented disturbance $\widetilde{\mathbf{D}}$ will also be convergent in finite time. This completes the proof.

Remark 3. In the design process of the disturbance observer, the upper boundary of the augmented disturbance should be known. In general, the design parameter should be chosen as a large positive constant to guarantee the design requirement of the proposed sliding mode disturbance observer.

3.2. Terminal Sliding Mode Control for MIMO Nonlinear System. In this section, the finite-time tracking control scheme for MIMO nonlinear system is proposed based on the terminal sliding mode. Utilizing the output of the disturbance observer as feedforward compensation, the designed terminal sliding mode control can guarantee finite-time convergence of the closed-loop signal in the system.

To derive the TSM controller, we need to define the tracking error:

$$
\mathbf{S}_{1}=\mathbf{x}_{1}-\mathbf{y}_{d}
$$

Time differentiation of $\mathbf{S}_{1}$ yields

$$
\begin{aligned}
& \dot{\mathbf{S}}_{1}=\dot{\mathbf{x}}_{1}-\dot{\mathbf{y}}_{d}=\mathbf{x}_{2}-\dot{\mathbf{y}}_{d} \\
& \ddot{\mathbf{S}}_{1}=\dot{\mathbf{x}}_{2}-\ddot{\mathbf{y}}_{d}=\mathbf{f}(\mathbf{x})+\mathbf{v}_{r}+\mathbf{D}-\ddot{\mathbf{y}}_{d} .
\end{aligned}
$$

Next, the terminal sliding mode surface is chosen as

$$
\mathbf{S}_{2}=\dot{\mathbf{S}}_{1}+\boldsymbol{\alpha} \mathbf{S}_{1}+\boldsymbol{\beta}_{1} \mathbf{S}_{1}^{p_{1} / q_{1}}+\mathbf{S}
$$

where $\mathbf{S}$ is defined in the disturbance observer, $0<p_{1}<q_{1}$, $\boldsymbol{\alpha}_{1}$ and $\boldsymbol{\beta}_{1}$ are positive definite diagonal matrices, and the other parameters are defined in the following form:

$$
\begin{aligned}
& \boldsymbol{\alpha}_{1}=\operatorname{diag}\left(\left[\begin{array}{llll}
\alpha_{1} & \alpha_{2} & \cdots & \alpha_{n}
\end{array}\right]\right), \\
& \boldsymbol{\beta}_{1}=\operatorname{diag}\left(\left[\begin{array}{llll}
\beta_{11} & \beta_{12} & \cdots & \beta_{1 n}
\end{array}\right]\right) .
\end{aligned}
$$

Invoking (18) and (19), the time derivative of the terminal sliding mode surface can be computed as

$$
\begin{aligned}
\dot{\mathbf{S}}_{2} & =\ddot{\mathbf{S}}_{1}+\boldsymbol{\alpha}_{1} \dot{\mathbf{S}}_{1}+\boldsymbol{\beta}_{1} \frac{d \mathbf{S}_{1}^{p_{1} / q_{1}}}{d t}+\dot{\mathbf{S}} \\
& =\mathbf{f}(\mathbf{x})+\mathbf{v}_{r}+\mathbf{D}-\ddot{\mathbf{y}}_{d}+\boldsymbol{\alpha}_{1} \dot{\mathbf{S}}_{1}+\boldsymbol{\beta}_{1} \frac{d \mathbf{S}_{1}^{p_{1} / q_{1}}}{d t}+\widetilde{\mathbf{D}} \\
& =\mathbf{f}(\mathbf{x})+\mathbf{v}_{r}-\ddot{\mathbf{y}}_{d}+\boldsymbol{\alpha}_{1} \dot{\mathbf{S}}_{1}+\boldsymbol{\beta}_{1} \frac{d \mathbf{S}_{1}^{p_{1} / q_{1}}}{d t}+\widehat{\mathbf{D}}
\end{aligned}
$$


Then, the terminal sliding mode control for MIMO nonlinear system is designed as

$$
\begin{aligned}
\mathbf{v}_{r}= & -\mathbf{f}(\mathbf{x})+\ddot{\mathbf{y}}_{d}-\boldsymbol{\alpha}_{1} \dot{\mathbf{S}}_{1}-\boldsymbol{\beta}_{1} \frac{d \mathbf{S}_{1}^{p_{1} / q_{1}}}{d t}-\widehat{\mathbf{D}}-\boldsymbol{\delta} \mathbf{S}_{2} \\
& -\boldsymbol{\mu} \mathbf{S}_{2}^{p_{2} / q_{2}}
\end{aligned}
$$

where $\boldsymbol{\delta}$ and $\boldsymbol{\mu}$ are positive definite diagonal matrices, $0<$ $p_{2}<q_{2}$.

Substituting (22) into (2) and (7), we can get the actual control command.

$$
\mathbf{u}= \begin{cases}\mathbf{u}_{\max } & \mathbf{G}(\mathbf{x})^{-1} \boldsymbol{\nu}_{r}>\mathbf{u}_{\max } \\ \mathbf{G}(\mathbf{x})^{-1} \boldsymbol{v}_{r} & -\mathbf{u}_{\min } \leq \mathbf{G}(\mathbf{x})^{-1} \boldsymbol{\nu}_{r} \leq \mathbf{u}_{\max } \\ -\mathbf{u}_{\min } & \mathbf{G}(\mathbf{x})^{-1} \boldsymbol{\nu}_{r}<-\mathbf{u}_{\min } .\end{cases}
$$

The above design procedure and analysis can be summarized in the following theorem.

Theorem 4. Consider the uncertain MIMO nonlinear system with external disturbance and asymmetric input saturation in (1). Assume that the full state information is available. If the finite-time disturbance observer is designed as in (9)-(12), all the states of the closed-loop system will converge to the equilibrium point under the proposed terminal sliding mode control in finite-time.

Proof. Consider the Lyapunov function candidate:

$$
V_{2}=\frac{1}{2} \mathbf{S}_{2}^{T} \mathbf{S}_{2}
$$

Substituting (23) into (22) results in

$$
\dot{\mathbf{S}}_{2}=-\boldsymbol{\delta} \mathbf{S}_{2}-\boldsymbol{\mu} \mathbf{S}_{2}^{p_{2} / q_{2}}
$$

Hence, the derivative of $V_{2}$ can be calculated as follows:

$$
\begin{aligned}
\dot{V}_{2} & =\mathbf{S}_{2}^{T} \dot{\boldsymbol{S}}_{2}=\mathbf{S}_{2}^{T}\left(-\boldsymbol{\delta} \mathbf{S}_{2}-\boldsymbol{\mu} \mathbf{S}_{2}^{p_{2} / q_{2}}\right) \\
& =-\sum_{i=1}^{n} \delta_{i} S_{2 i}^{2}-\sum_{i=1}^{n} \mu_{i} S_{2 i}^{\left(p_{2}+q_{2}\right) / q_{2}} \\
& =-2 \lambda_{\min }(\boldsymbol{\delta}) V_{2}-2^{\left(p_{2}+q_{2}\right) / 2 q_{2}} \lambda_{\min }(\boldsymbol{\mu}) V_{2}^{\left(p_{2}+q_{2}\right) / 2 q_{2}}
\end{aligned}
$$

According to (27) and Lemma 1, it can be concluded that the terminal sliding mode surface $\mathbf{S}_{2}$ will converge to zero in finite time. And the convergence time of $\mathbf{S}_{2}$ can be calculated as follows:

$$
\begin{aligned}
t_{2}= & \frac{1}{2 \lambda_{\min }(\boldsymbol{\delta})\left(1+\left(p_{2}+q_{2}\right) / 2 q_{2}\right)} \\
& \cdot \ln \frac{2 \lambda_{\min }(\boldsymbol{\delta}) V_{2}^{\left(q_{2}-p_{2}\right) / 2 q_{2}}(0)+2^{\left(p_{2}+q_{2}\right) / q_{2}} \lambda_{\min }(\boldsymbol{\mu})}{2^{\left(p_{2}+q_{2}\right) / q_{2}} \lambda_{\min }(\boldsymbol{\mu})}
\end{aligned}
$$

In fixed interval $t=\max \left\{t_{1}, t_{2}\right\}$, both $\mathbf{S}_{2}$ and $\mathbf{S}$ are convergent to zero. Moreover, the convergence of $\boldsymbol{S}_{1}$ can also be derived from (20). Then, $\mathbf{S}, \mathbf{S}_{1}$, and $\mathbf{S}_{2}$ are finite-time convergent. That is, the estimated disturbance will converge to the actual augmented disturbance and the trajectory of the system will converge to the desired trajectory. Thus, all closed-loop signals converge to the equilibrium point in the finite time. This completes the proof.

Remark 5. From (8), the effect of external disturbance and asymmetric input saturation is treated as an augmented disturbance, which is estimated by the finite-time disturbance observer. Through feedforward compensation, the observerbased terminal sliding mode controller can make the trajectory of the closed-loop system converge to the desired trajectory in finite time regardless of the unknown input saturation and external disturbance.

\section{Simulation Results}

In this section, simulation results are given to validate the effectiveness of the proposed finite time control scheme for a surface vessel under two different situations: without input saturation and with input saturation. In the first situation, finite time convergence of the closed-loop signals is illustrated. In the second situation, chattering elimination and better control performance of our scheme are demonstrated through comparing with the existing finite-time control method.

The classic 3-DOF mathematic vessel model is given by the following equations [47]:

$$
\begin{aligned}
\dot{\boldsymbol{\eta}} & =\mathbf{R}(\psi) \mathbf{v}, \\
\mathbf{M} \dot{\mathbf{v}}+\mathbf{D}(\mathbf{v}) \mathbf{v} & =\boldsymbol{\tau}+\boldsymbol{\omega} .
\end{aligned}
$$

Here $\boldsymbol{\eta}=\left[\begin{array}{lll}n & e & \psi\end{array}\right]^{T}$ is the position and orientation of the vessel with respect to an inertial reference coordinate system, and $\mathbf{v}=\left[\begin{array}{lll}u & v & r\end{array}\right]^{T}$ is the vector of velocities given in the body-fixed coordinate system. $\boldsymbol{\tau}$ is the control input and $\boldsymbol{\omega}$ is the environmental disturbance. $\mathbf{M}$ and $\mathbf{D}(\mathbf{v})$ are the system inertia matrix and damping matrix, respectively. $\mathbf{R}(\psi)$ is a transformation matrix between the inertial and body-fixed coordinate frames, with

$$
\mathbf{R}(\psi)=\left(\begin{array}{ccc}
\cos \psi & -\sin \psi & 0 \\
\sin \psi & \cos \psi & 0 \\
0 & 0 & 1
\end{array}\right)
$$

The parameters of the vessel and the external environment interference in this paper are shown in Table 1 , where $m, L$, and $B$ denote the mass, length, and breadth of the vessel, respectively. $W \_s, W \_a, C \_s$, and $C \_a$ are the wind parameters and current parameters respectively. In this simulation, just the wind and current are considered, and their mathematical 
TABLE 1: Vessel and environmental parameters.

\begin{tabular}{lc}
\hline Parameter & Value \\
\hline$m$ & $6.3 e 7(\mathrm{~kg})$ \\
$L$ & $185(\mathrm{~m})$ \\
$B$ & $39.2(\mathrm{~m})$ \\
$W_{-} s$ & $17.1(\mathrm{~m} / \mathrm{s})$ \\
$W_{\_} a$ & $\pi / 3(\mathrm{rad})$ \\
$C \_s$ & $0.771(\mathrm{~m} / \mathrm{s})$ \\
$C \_a$ & $\pi / 12(\mathrm{rad})$ \\
\hline
\end{tabular}

models are referred to [43]. Furthermore, other parameters of the vessel model are chosen as

$\mathbf{M}$

$$
\begin{aligned}
& =\left[\begin{array}{ccc}
6.6073 \times 10^{7} & 0 & 0 \\
0 & 8.9985 \times 10^{7} & -3.7964 \times 10^{7} \\
0 & -3.7964 \times 10^{7} & 2.7050 \times 10^{11}
\end{array}\right], \\
& \mathbf{D}=\left[\begin{array}{ccc}
0 & 0 & 0 \\
0 & 2.7468 \times 10^{5} & -6.2368 \times 10^{6} \\
0 & -6.2368 \times 10^{6} & 1.4528 \times 10^{9}
\end{array}\right] .
\end{aligned}
$$

To facilitate the control design process, the vessel model is rewritten in the following form:

$$
\begin{aligned}
& \dot{\mathbf{x}}_{1}=\mathbf{x}_{2}, \\
& \dot{\mathbf{x}}_{2}=\mathbf{f}(\mathbf{x})+\mathbf{g}(\mathbf{x}) \boldsymbol{\tau}+\mathbf{d},
\end{aligned}
$$

where

$$
\begin{aligned}
& \mathbf{x}_{1}=\boldsymbol{\eta}, \\
& \mathbf{x}_{2}=\dot{\boldsymbol{\eta}}, \\
& \mathbf{d}=\left(\mathbf{R}^{-T}(\psi) \mathbf{M} \mathbf{R}^{-1}(\psi)\right)^{-1} \mathbf{R}^{-T}(\psi) \boldsymbol{\omega}, \\
& \mathbf{f}(\mathbf{x}) \\
& \quad=-\left(\mathbf{R}^{-T}(\psi) \mathbf{M R}^{-1}(\psi)\right)^{-1}\left(\mathbf{R}^{-T}(\psi) \mathbf{D} \mathbf{R}^{-1}(\psi)\right) \dot{\boldsymbol{\eta}}, \\
& \mathbf{g}(\mathbf{x})=\left(\mathbf{R}^{-T}(\psi) \mathbf{M} \mathbf{R}^{-1}(\psi)\right)^{-1} \mathbf{R}^{-T}(\psi), \\
& \mathbf{d}=\mathbf{g}(\mathbf{x}) \boldsymbol{\omega} .
\end{aligned}
$$

In the following numerical simulation, we use the proposed controller to track a circle:

$$
\begin{aligned}
& \mathbf{y}_{d} \\
& =\left[\begin{array}{lll}
150 \times \sin (0.0025 \times t) & 150 \times \cos (0.0025 \times t) & 0.5
\end{array}\right]^{T} .
\end{aligned}
$$

The initial states of the system are set to be

$$
\begin{aligned}
& \mathbf{x}_{1}(0)=\boldsymbol{\eta}(0)=\left[\begin{array}{lll}
-1 & 149 & 0.2
\end{array}\right]^{T}, \\
& \mathbf{x}_{2}(0)=\mathbf{z}(0)=\left[\begin{array}{lll}
0 & 0 & 0
\end{array}\right]^{T} .
\end{aligned}
$$

4.1. Without Considering Input Saturation for Surface Vessel. In this case, only the unmeasured external disturbances are considered and the control input control input is assumed to be unconstrained. According to the control design process, the finite-time disturbance observer and the terminal sliding mode controller are given by

$$
\begin{aligned}
& \mathbf{S}=\mathbf{z}-\mathbf{x}_{2}, \\
& \dot{\mathbf{z}}=-\mathbf{K S}-\boldsymbol{\beta} \operatorname{sgn}(\mathbf{S})-\boldsymbol{\varepsilon} \mathbf{S}^{p / q}-\mathbf{F}(\mathbf{x}) \operatorname{sgn}(\mathbf{S})+\mathbf{v}_{r}, \\
& \mathbf{S}_{1}=\mathbf{x}_{1}-\mathbf{y}_{d}, \\
& \mathbf{S}_{2}=\dot{\mathbf{S}}_{1}+\boldsymbol{\alpha}_{1} \mathbf{S}_{1}+\boldsymbol{\beta}_{1} \mathbf{S}_{1}^{p_{1} / q_{1}}+\mathbf{S}, \\
& \widehat{\mathbf{d}}=-\mathbf{K S}-\boldsymbol{\beta} \operatorname{sgn}(\mathbf{S})-\boldsymbol{\varepsilon} \mathbf{S}^{p / q}-\mathbf{F}(\mathbf{x}) \operatorname{sgn}(\mathbf{S})-\mathbf{f}(\mathbf{x}), \\
& \boldsymbol{\tau}=\mathbf{g}(\mathbf{x})^{-1}\left[-\mathbf{f}(\mathbf{x})+\ddot{\mathbf{y}}_{d}-\boldsymbol{\alpha}_{1} \dot{\mathbf{S}}_{1}-\boldsymbol{\beta}_{1} \frac{d \mathbf{S}_{1}^{p_{1} / q_{1}}}{d t}-\widehat{\mathbf{d}}\right. \\
& \left.-\boldsymbol{\delta} \mathbf{S}_{2}-\boldsymbol{\mu} \mathbf{S}_{2}^{p_{2} / q_{2}}\right],
\end{aligned}
$$

where $\widehat{\mathbf{d}}$ is the estimation of $\mathbf{d}$ and control parameters are chosen as

$$
\begin{aligned}
\mathbf{K} & =\mathbf{I}_{3 \times 3}, \\
\boldsymbol{\beta} & =0.02 \times \mathbf{I}_{3 \times 3}, \\
\boldsymbol{\varepsilon} & =0.25 \times \mathbf{I}_{3 \times 3}, \\
\boldsymbol{\alpha}_{1} & =37 \times \mathbf{I}_{3 \times 3}, \\
\boldsymbol{\beta}_{1} & =0.5 \times \mathbf{I}_{3 \times 3}, \\
\boldsymbol{\delta} & =60 \times \mathbf{I}_{3 \times 3}, \\
\boldsymbol{\mu} & =0.8 \times \mathbf{I}_{3 \times 3}, \\
p & =5, \\
q & =9, \\
p_{1} & =p_{2}=5, \\
q_{1} & =q_{2}=7 .
\end{aligned}
$$

Under the proposed observer-based finite time control, from Figures 2 and 3, we can observe that the disturbance estimate error and tracking error quickly converge to zero in a short finite time in the presence of the time-varying external disturbance. The sliding mode and the control input are shown in Figures 4 and 5. Furthermore, the convergence time of the closed-loop system calculated from the theoretical equations (17) and (28) is $t=0.876 \mathrm{~s}$. From Figures 2-4, it is clear to conclude that all the signals of the closedloop system are convergent to zero within $0.876 \mathrm{~s}$, which validate the correctness of the control scheme. Figure 5 shows that the control input is bounded and convergent. Based on the simulation results, we can obtain that the finite-time convergence performance of the method presented in this paper is valid for the uncertain nonlinear system with the time-varying external disturbance. 

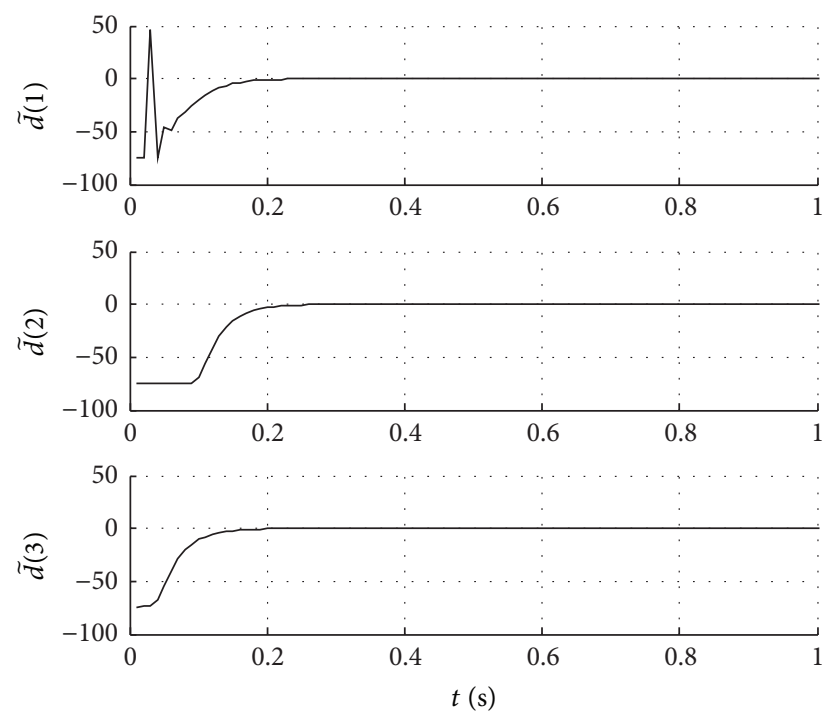

FIGURE 2: The disturbance estimate error without input saturation.
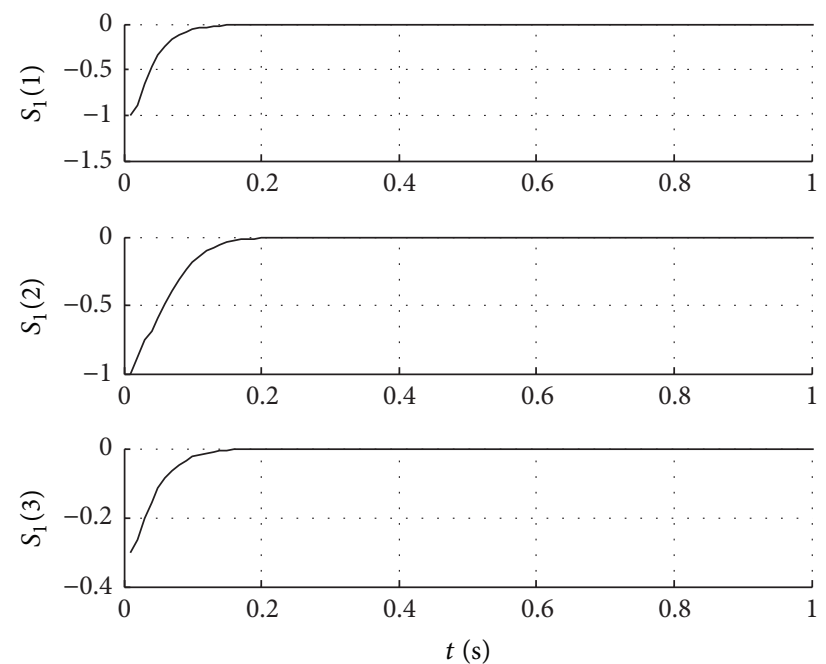

FIGURE 3: Tracking error without input saturation.
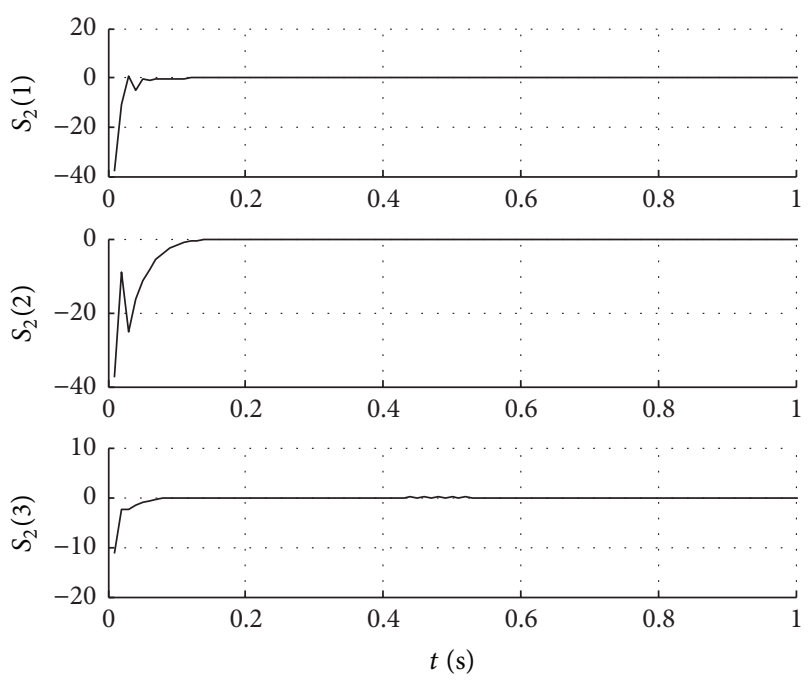

FIgURE 4: Sliding mode without input saturation.
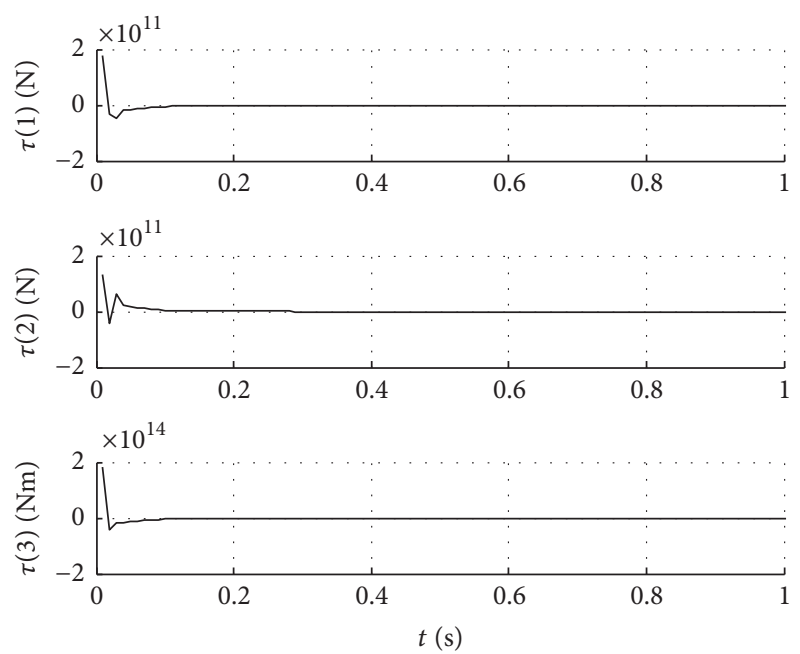

FIGURE 5: Control input without input saturation.

4.2. Considering Asymmetric Input Saturation for Surface Vessel. In this case, the input saturation is set to be

$$
\begin{aligned}
& \boldsymbol{\tau}_{\max }=\left[\begin{array}{lll}
4 \times 10^{7} & 4 \times 10^{7} & 2 \times 10^{10}
\end{array}\right]^{T}, \\
& \boldsymbol{\tau}_{\min }=\left[\begin{array}{lll}
-3 \times 10^{7} & -2 \times 10^{7} & -1.9 \times 10^{10}
\end{array}\right]^{T} .
\end{aligned}
$$

The disturbance-observer-based finite-time controller is designed as

$$
\begin{aligned}
& \mathbf{S}_{1}=\mathbf{x}_{1}-\mathbf{y}_{d}, \\
& \mathbf{S}_{2}=\dot{\mathbf{S}}_{1}+\boldsymbol{\alpha}_{1} \mathbf{S}_{1}+\boldsymbol{\beta}_{1} \mathbf{S}_{1}^{p_{1} / q_{1}}+\mathbf{S} \\
& \widehat{\mathbf{D}}=-\mathbf{K S}-\boldsymbol{\beta} \operatorname{sgn}(\mathbf{S})-\boldsymbol{\varepsilon} \mathbf{S}^{p / q}-\mathbf{F}(\mathbf{x}) \operatorname{sgn}(\mathbf{S})-\mathbf{f}(\mathbf{x}), \\
& \boldsymbol{\nu}=\mathbf{g}(\mathbf{x})^{-1}\left[-\mathbf{f}(\mathbf{x})+\ddot{\mathbf{y}}_{d}-\boldsymbol{\alpha}_{1} \dot{\mathbf{S}}_{1}-\boldsymbol{\beta}_{1} \frac{d \mathbf{S}_{1}^{p_{1} / q_{1}}}{d t}-\widehat{\mathbf{D}}\right. \\
& \left.-\boldsymbol{\delta} \mathbf{S}_{2}-\boldsymbol{\mu} \mathbf{S}_{2}^{p_{2} / q_{2}}\right] \\
& \boldsymbol{\tau}= \begin{cases}\boldsymbol{\tau}_{\max } & \boldsymbol{\nu}>\boldsymbol{\tau}_{\max } \\
\boldsymbol{\nu} & \boldsymbol{\tau}_{\min } \leq \boldsymbol{\nu} \leq \boldsymbol{\tau}_{\max } \\
\boldsymbol{\tau}_{\min } & \boldsymbol{\nu}<\boldsymbol{\tau}_{\min } .\end{cases}
\end{aligned}
$$

In this case, we select the same control parameters as before. To show the better control performance of our finite-time control scheme, we make comparison with the disturbance-observer-based sliding mode control proposed in [48]. From the tracking trajectories and tracking error presented in Figures 6 and 7, it can be seen that our method has better tracking performance. Figure 8 shows that sliding mode jitter can be eliminated effectively by the proposed scheme, which in turn illustrates the more smooth control input shown in Figure 9. Despite the presence of asymmetric input saturations and time-varying external disturbances, the 


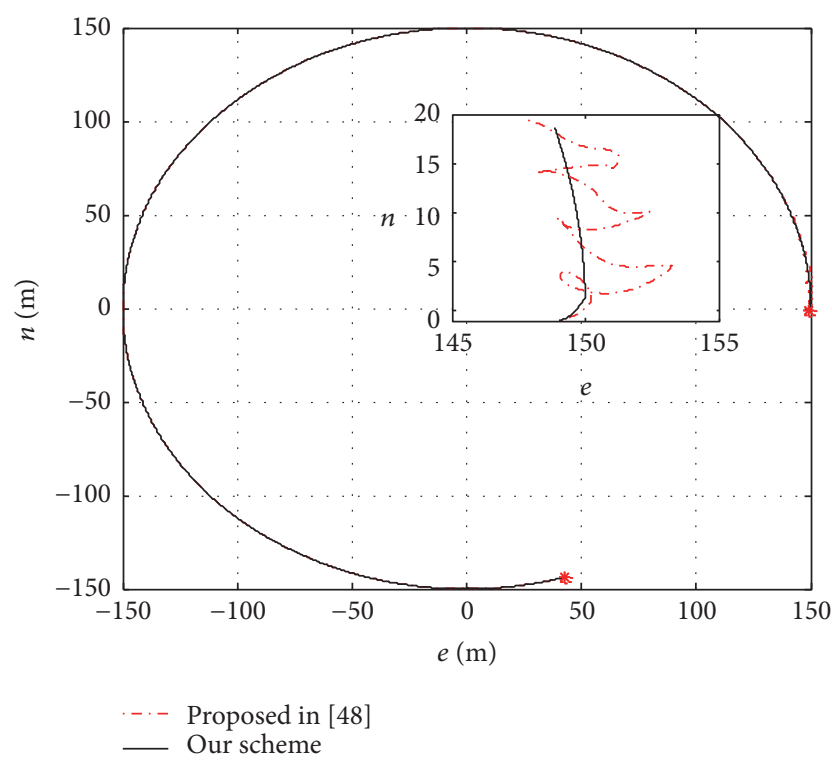

FIGURE 6: Tracking trajectories with input saturation.
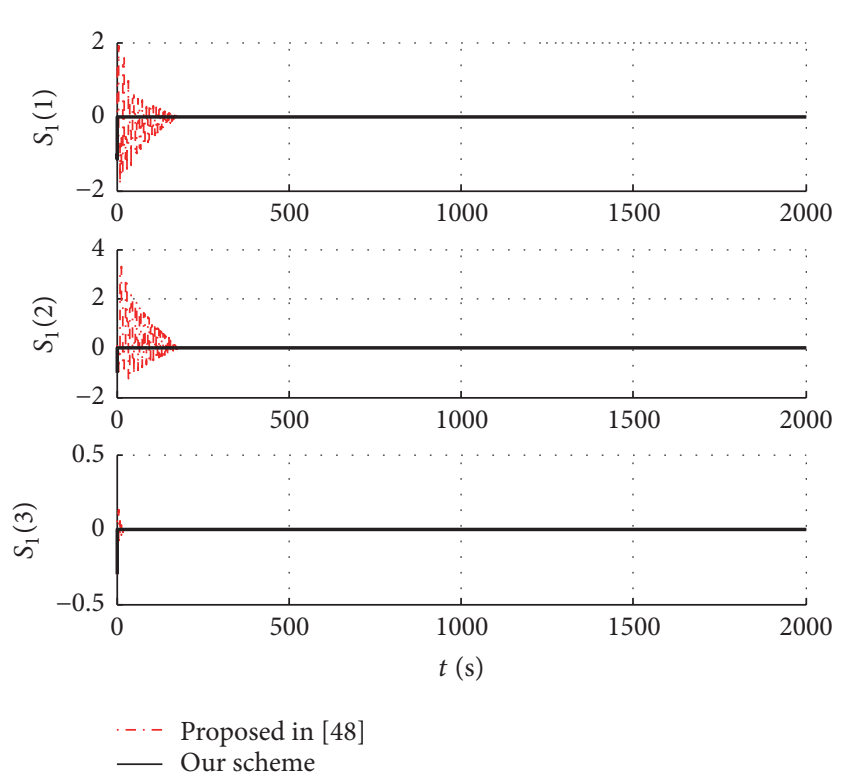

FIGURE 7: Tracking error with input saturation.

tracking performance is still satisfactory and the tracking error converges to zero.

Based on the above simulation, we can conclude that the proposed observer-based finite-time controller can mitigate the chattering phenomena due to the input saturation.

\section{Conclusion}

In this paper, the disturbance-observer-based finite-time tracking control has been proposed for a class of MIMO uncertain nonlinear systems. To improve the ability of the disturbance attenuation and system performance robustness, the
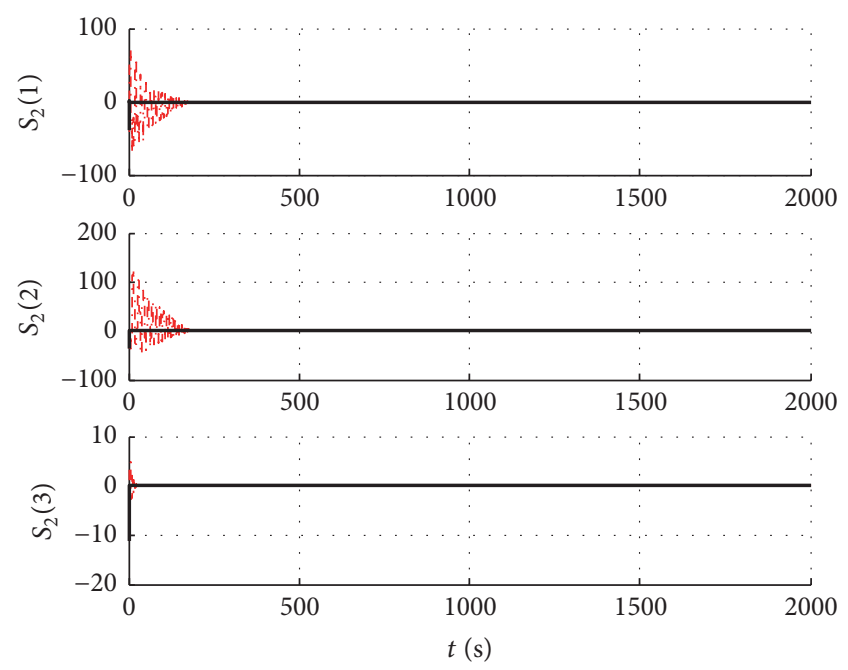

-. Proposed in [48]

— Our scheme

FIGURE 8: Sliding mode with input saturation.
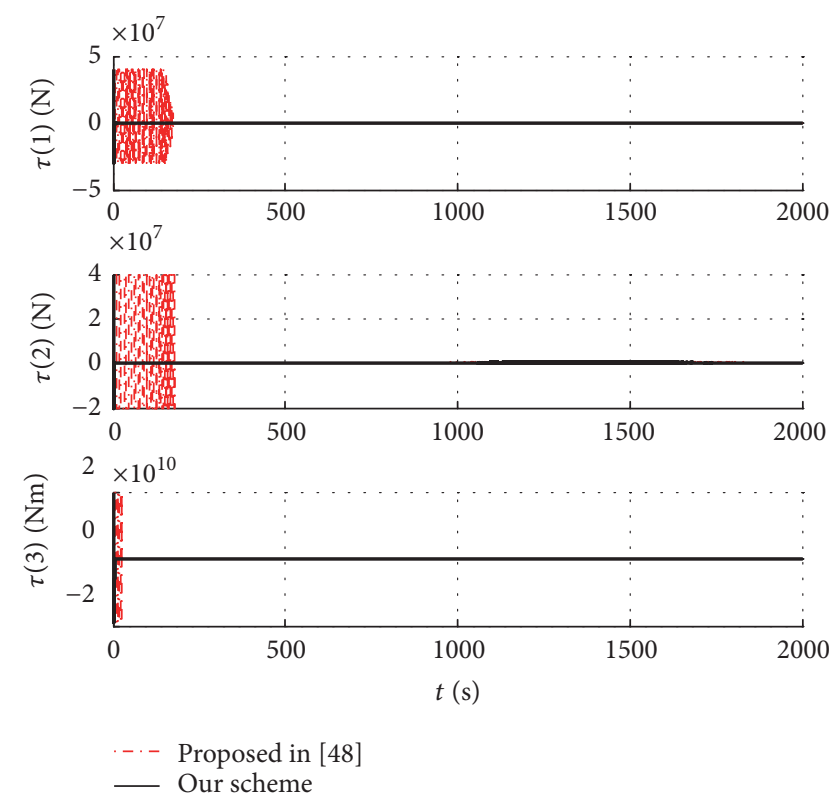

FIGURE 9: Control input with input saturation.

terminal sliding mode disturbance observer has been developed to approximate the system disturbance. Based on the output of the disturbance observer, the disturbance-observerbased finite-time tracking control has been presented for the uncertain nonlinear system with the nonsymmetric input saturation and the time-varying external disturbance. Then, the chattering phenomena caused by the external and input saturation can be eliminated. The stability of the closed-loop system has been proved using rigorous Lyapunov analysis. Finally, simulation results have been used to illustrate the effectiveness of the proposed robust terminal sliding mode tracking control scheme. 


\section{Conflicts of Interest}

The authors declare that there are no conflicts of interest regarding the publication of this paper.

\section{Acknowledgments}

This paper is supported by special funds for the Comprehensive Pilot Project of Discipline Construction in College of Automation, Harbin Engineering University.

\section{References}

[1] S. P. Bhat and D. S. Bernstein, "Finite-time stability of continuous autonomous systems," SIAM Journal on Control and Optimization, vol. 38, no. 3, pp. 751-766, 2000.

[2] F. Amato, R. Ambrosino, M. Ariola, C. Cosentino, and G. De Tommasi, Finite-time stability and control, vol. 453 of Lecture Notes in Control and Information Sciences, Springer, London, UK, 2014

[3] Y. Dong and F. Yang, "Finite-time stability and boundedness of switched nonlinear time-delay systems under state-dependent switching," Complexity, vol. 21, no. 2, pp. 267-275, 2015.

[4] W. Xiang and J. Xiao, " $H_{\infty}$ finite-time control for switched nonlinear discrete-time systems with norm-bounded disturbance," Journal of the Franklin Institute, vol. 348, no. 2, pp. 331-352, 2011.

[5] Y. Su and C. Zheng, "Robust finite-time output feedback control of perturbed double integrator," Automatica, vol. 60, pp. 86-91, 2015.

[6] F. Amato, R. Ambrosino, C. Cosentino, and G. De Tommasi, "Input-output finite time stabilization of linear systems," Automatica, vol. 46, no. 9, pp. 1558-1562, 2010.

[7] M. T. Angulo, L. Fridman, and A. Levant, "Output-feedback finite-time stabilization of disturbed LTI systems," Automatica, vol. 48, no. 4, pp. 606-611, 2012.

[8] S. Bhat and D. S. Bernstein, "Continuous, bounded, finitetime stabilization of the translational and rotational double integrators," Clinical \& Experimental Dermatology, vol. 30, no. 3, pp. 272-276, 2005.

[9] S. P. Bhat and D. S. Bernstein, "Geometric homogeneity with applications to finite-time stability," Mathematics of Control, Signals, and Systems, vol. 17, no. 2, pp. 101-127, 2005.

[10] A. Levant, "Universal single-input-single-output (SISO) sliding-mode controllers with finite-time convergence," Institute of Electrical and Electronics Engineers. Transactions on Automatic Control, vol. 46, no. 9, pp. 1447-1451, 2001.

[11] I. Nagesh and C. Edwards, "A multivariable super-twisting sliding mode approach," Automatica, vol. 50, no. 3, pp. 984-988, 2014.

[12] M. Zhihong, A. P. Paplinski, and H. R. Wu, "A robust MIMO terminal sliding mode control scheme for rigid robotic manipulators," Institute of Electrical and Electronics Engineers. Transactions on Automatic Control, vol. 39, no. 12, pp. 2464-2469, 1994.

[13] X. Yu and M. Zhihong, "Fast terminal sliding-mode control design for nonlinear dynamical systems," IEEE Transactions on Circuits and Systems I: Fundamental Theory and Applications, vol. 49, no. 2, pp. 261-264, 2002.

[14] Y. Feng, X. Yu, and Z. Man, "Non-singular terminal sliding mode control of rigid manipulators," Automatica, vol. 38, no. 12, pp. 2159-2167, 2002.
[15] Y. Wu, X. Yu, and Z. Man, "Terminal sliding mode control design for uncertain dynamic systems," Systems and Control Letters, vol. 34, no. 5, pp. 281-287, 1998.

[16] A. Al-Ghanimi, J. Zheng, and Z. Man, "Robust and fast nonsingular terminal sliding mode control for piezoelectric actuators," IET Control Theory \& Applications, vol. 9, no. 18, pp. 26782687, 2015.

[17] S. E. Li and K. Deng, "Recent advances in nonsingular terminal sliding mode control method," Lecture Notes in Control and Information Sciences, vol. 452, pp. 79-97, 2014.

[18] H.-X. Zhang, J.-S. Fan, F. Meng et al., "A new double power reaching law for sliding mode control," Control and Decision, vol. 28, no. 2, pp. 289-293, 2013.

[19] H. Li, L. Dou, and Z. Su, "Adaptive nonsingular fast terminal sliding mode control for electromechanical actuator," International Journal of Systems Science, vol. 44, no. 3, pp. 401-415, 2013.

[20] Y. Zhang, G.-F. Ma, Y.-N. Guo, and T.-Y. Zeng, "A multi power reaching law of sliding mode control design and analysis," Zidonghua Xuebao/Acta Automatica Sinica, vol. 42, no. 3, pp. 466-472, 2016.

[21] D. Y. Shi, S. Q. Liao, Y. C. Lin et al., "Fractional terminal sliding mode control design for a class of dynamical systems with uncertainty," Communications in Nonlinear Science \& Numerical Simulation, vol. 17, no. 1, pp. 367-377, 2012.

[22] N. Zhou and Y. Xia, "Coordination control design for formation reconfiguration of multiple spacecraft," IET Control Theory \& Applications, vol. 9, no. 15, pp. 2222-2231, 2015.

[23] J. Zheng, H. Wang, Z. Man, J. Jin, and M. Fu, "Robust Motion Control of a Linear Motor Positioner Using Fast Nonsingular Terminal Sliding Mode," IEEE/ASME Transactions on Mechatronics, pp. 1-10, 2014.

[24] M. Jin, J. Lee, and K. K. Ahn, "Continuous nonsingular terminal sliding-mode control of shape memory alloy actuators using time delay estimation," IEEE/ASME Transactions on Mechatronics, vol. 20, no. 2, pp. 899-909, 2015.

[25] S. Laghrouche, F. Plestan, and A. Glumineau, Brief Paper: Higher Order Sliding Mode Control Based on Integral Sliding Mode, Pergamon Press, Inc., Oxford, UK, 2007.

[26] F. Dinuzzo and A. Ferrara, "Higher order sliding mode controllers with optimal reaching," Institute of Electrical and Electronics Engineers. Transactions on Automatic Control, vol. 54, no. 9, pp. 2126-2136, 2009.

[27] E. Kim, "A fuzzy disturbance observer and its application to control," IEEE Transactions on Fuzzy Systems, vol. 10, no. 1, pp. 77-84, 2002.

[28] A. Mohammadi, M. Tavakoli, H. J. Marquez, and F. Hashemzadeh, "Nonlinear disturbance observer design for robotic manipulators," Control Engineering Practice, vol. 21, no. 3, pp. 253-267, 2013.

[29] H. Su and G.-Y. Tang, "Observer-based approximate optimal tracking control for time-delay systems with external disturbances," International Journal of Systems Science, vol. 47, no. 12, pp. 1-10, 2015.

[30] J. Q. Han, "From PID to active disturbance rejection control," IEEE Transactions on Industrial Electronics, vol. 56, no. 3, pp. 900-906, 2009.

[31] B.-Z. Guo and Z.-L. Zhao, "On the convergence of an extended state observer for nonlinear systems with uncertainty," Systems \& Control Letters, vol. 60, no. 6, pp. 420-430, 2011.

[32] A. A. Godbole, J. P. Kolhe, and S. E. Talole, "Performance analysis of generalized extended state observer in tackling sinusoidal 
disturbances," IEEE Transactions on Control Systems Technology, vol. 21, no. 6, pp. 2212-2223, 2013.

[33] H. Kim, J. Son, and J. Lee, "A high-speed sliding-mode observer for the sensorless speed control of a PMSM," IEEE Transactions on Industrial Electronics, vol. 58, no. 9, pp. 4069-4077, 2011.

[34] S. M. Kazraji, R. B. Soflayi, and M. B. B. Sharifian, "Sliding-Mode Observer for Speed and Position Sensorless Control of LinearPMSM," Electrical Control \& Communication Engineering, vol. 5, no. 1, pp. 20-26, 2014.

[35] M. Chen, Q.-X. Wu, and R.-X. Cui, "Terminal sliding mode tracking control for a class of SISO uncertain nonlinear systems," ISA Transactions, vol. 52, no. 2, pp. 198-206, 2013.

[36] S. Li and X. Wang, "Finite-time consensus and collision avoidance control algorithms for multiple AUVs," Automatica, vol. 49, no. 11, pp. 3359-3367, 2013.

[37] M. Chen, S. S. Ge, and B. V. E. How, "Robust adaptive neural network control for a class of uncertain MIMO nonlinear systems with input nonlinearities," IEEE Transactions on Neural Networks, vol. 21, no. 5, pp. 796-812, 2010.

[38] Y.-Y. Cao and Z. L. Lin, "Robust stability analysis and fuzzyscheduling control for nonlinear systems subject to actuator saturation," IEEE Transactions on Fuzzy Systems, vol. 11, no. 1, pp. 57-67, 2003.

[39] Y.-S. Zhong, "Globally stable adaptive system design for minimum phase SISO plants with input saturation," Automatica, vol. 41, no. 9, pp. 1539-1547, 2005.

[40] J. R. Azinheira and A. Moutinho, "Hover control of an UAV with backstepping design including input saturations," IEEE Transactions on Control Systems Technology, vol. 16, no. 3, pp. 517-526, 2008.

[41] M. Chen and J. Yu, "Adaptive dynamic surface control of NSVs with input saturation using a disturbance observer," Chinese Journal of Aeronautics, vol. 16, no. 3, pp. 853-864, 2015.

[42] J. Li, T. Li, and Y. Li, "NN-based adaptive dynamic surface control for a class of nonlinear systems with input saturation," in Proceedings of the 7th IEEE Conference on Industrial Electronics and Applications (ICIEA '12), pp. 570-575, IEEE, Singapore, July 2012.

[43] T. Li, R. Li, and J. Li, "Decentralized adaptive neural control of nonlinear interconnected large-scale systems with unknown time delays and input saturation," Neurocomputing, vol. 74, no. 14-15, pp. 2277-2283, 2011.

[44] R. Shahnazi, "Observer-based adaptive interval type-2 fuzzy control of uncertain MIMO nonlinear systems with unknown asymmetric saturation actuators," Neurocomputing, vol. 171, pp. 1053-1065, 2016.

[45] M. Zhihong and X. H. Yu, "Terminal sliding mode control of MIMO linear systems," IEEE Transactions on Circuits and Systems. I. Fundamental Theory and Applications, vol. 44, no. 11, pp. 1065-1070, 1997.

[46] S. Yu, X. Yu, B. Shirinzadeh, and Z. Man, "Continuous finitetime control for robotic manipulators with terminal sliding mode," Automatica, vol. 41, no. 11, pp. 1957-1964, 2005.

[47] T. I. Fossen, Handbook of Marine Craft Hydrodynamics and Motion Control, John Wiley \& Sons, West Sussex, UK, 2011.

[48] M. Chen and W.-H. Chen, "Sliding mode control for a class of uncertain nonlinear system based on disturbance observer," International Journal of Adaptive Control and Signal Processing, vol. 24, no. 1, pp. 51-64, 2010. 


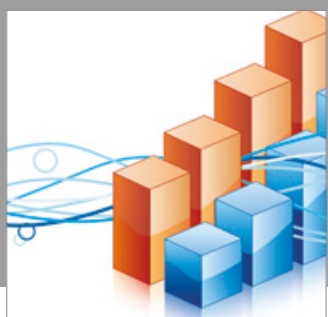

Advances in

Operations Research

vatersals

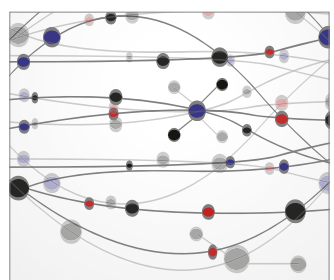

\section{The Scientific} World Journal
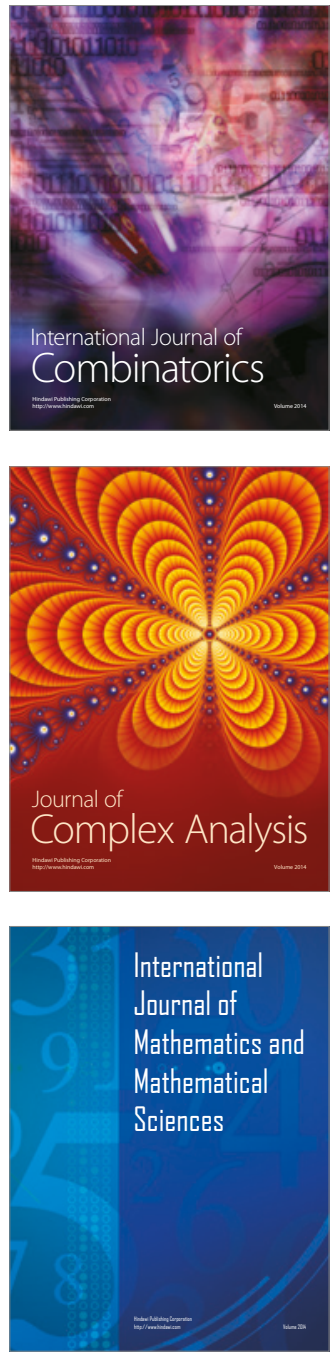
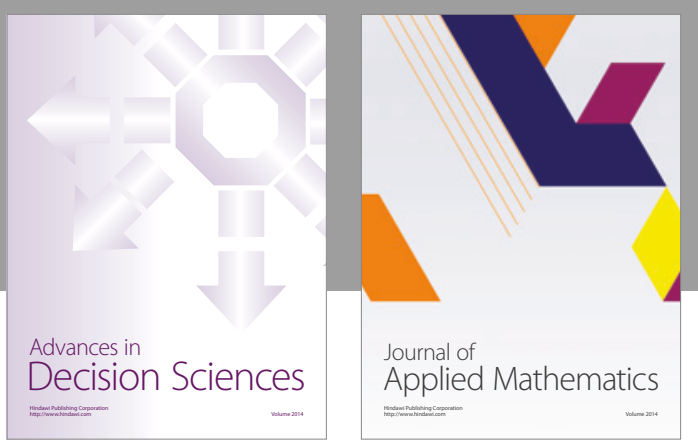

Algebra

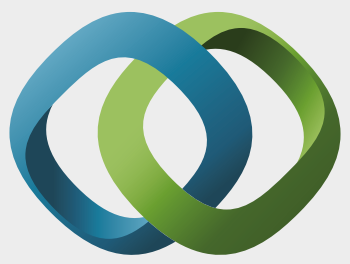

\section{Hindawi}

Submit your manuscripts at

https://www.hindawi.com
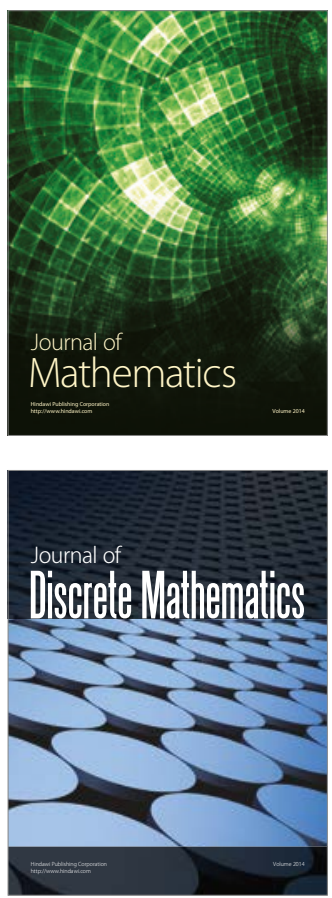

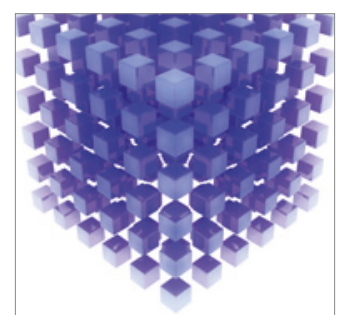

Mathematical Problems in Engineering
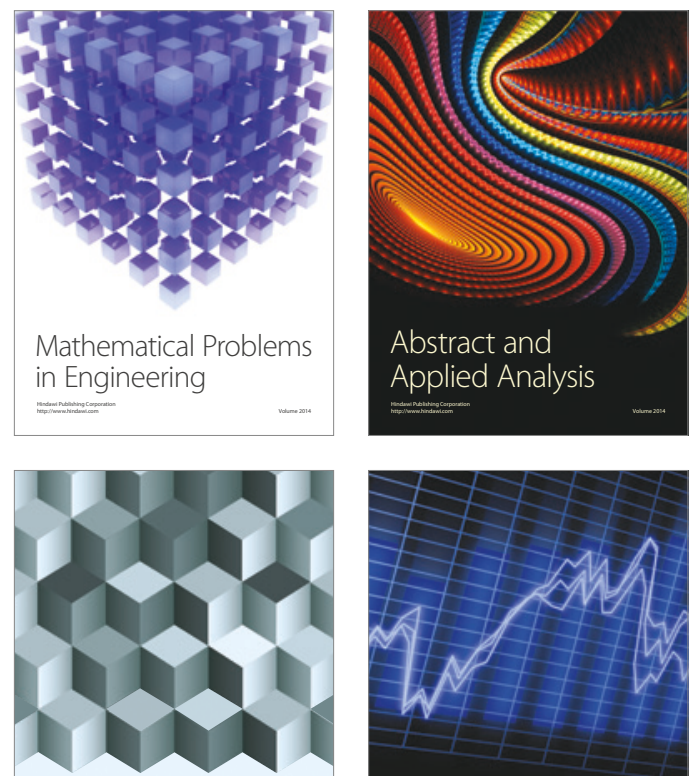

Journal of

Function Spaces

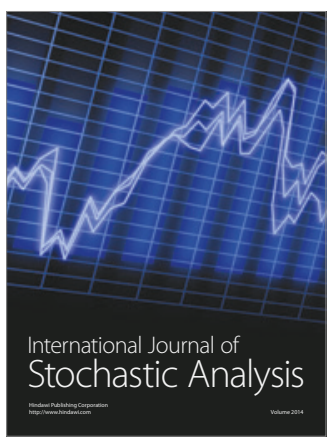

Probability and Statistics
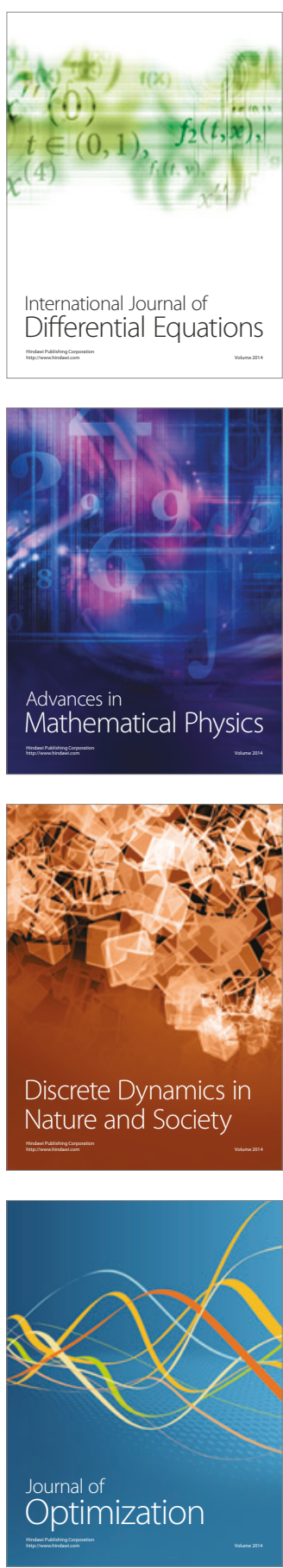